\title{
Computers and Creativity
}

\author{
Bipin Indurkhya \\ International Institute of Information Technology \\ Gachibowli, Hyderabad-500 019, INDIA \\ email:bipin@iiit.ac.in
}

\begin{abstract}
How can computers be used to model creativity? We address this issue in this article by starting with an examination of the two main cognitive mechanisms of human creativity. We argue that a primary hurdle facing human creativity is in trying to step outside of our habituated conceptual associations. However, since computers do not have such associations, we argue that in some important sense they are naturally predisposed towards creativity. Taking this viewpoint, we outline an approach to incorporating both the cognitive mechanism of creativity in a computational system.
\end{abstract}

\section{Introduction}

Computers by their very nature are inherently algorithmic; and often the mechanical nature of algorithms is considered an anathema to creativity. How can any creative insight result from following a predetermined set of instructions in a blind and seemingly mindless manner? This question is the main focus of this article.

The article is organized as follows. In the next section we elaborate upon what exactly we mean by creativity, and illustrate it with a few examples. In Section 3, we identify two main cognitive mechanisms of creativity that have been proposed by scholars who have studied creativity and have sometimes tried to teach people how to increase their creativity. Following that, in Section 4, we will examine how these mechanisms might be modeled computationally. Finally, in Section 5, we will highlight the main conclusions of this article and point to the future research issues.

\section{What do we mean by 'Creativity': Some examples}

\subsection{A Working Definition of Creativity}

For the purpose of this article, we are adopting the following as a working definition of 'creativity': A cognitive act is deemed creative if it provides a new perspective or new information about an object, situation or phenomenon. Though it may sound quite intuitive to some readers, it does have some implications that we should emphasize from the outset, before we start talking about the cognitive mechanisms of creativity and how they might be modeled.

First of all, the label 'creativity' is applied to individual cognitive acts, and not to the ability of some cognitive agent. Thus, our sense of creativity should not be confused with 'intelligence'. While it may be possible to correlate creative potential of a cognitive agent with its intelligence, we feel that incorporating this correlation into the definition of creativity distracts one from focusing on the creativity of a cognitive act, and puts too much emphasis on the creative potential of an 


\section{Bipin Indurkhya}

agent.

Secondly. we disregard the utility of the new perspective or information that results from a creative cognitive act. Obviously, in many contexts such as problem solving or art and poetry, this utility is an important consideration. However, at this initial foray into the mechanisms of creativity and how they might be modeled computationally, we feel that this issue is better left aside.

Finally, we make the assumption that the kind of creativity we are interested in exploring is something for which every cognitive agent has a potential. Thus, we consider quite relevant the works of those scholars who seek to teach people methods to enhance their creativity. Another corollary of this assumption is that as far as computational systems can be considered cognitive agents, it follows that they should be able to display creativity too.

\subsection{Creativity in Perceptual Acts}

It is perhaps best to illustrate our notion of creativity by means of a few examples. Let us first take a couple of examples of creativity in perception. Consider the geometric form shown in Figure 1. How would you describe this figure? What features do you see in it?

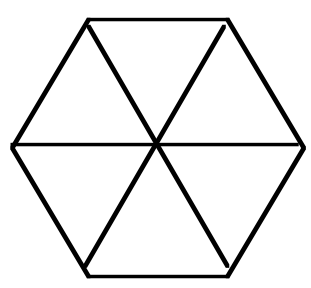

Fig. 1. Consider the figure above, and think about what perceptual features it might have.

Now consider the proportional analogy relation ' $\mathrm{A}$ is to $\mathrm{B}$ as $\mathrm{C}$ is to $\mathrm{D}$ ' shown in Figure 2, in which the term B is the same as Figure 1. Can you understand this analogy? If you do, then do you see parallelograms in Figure 1 now? This example illustrates how context can bring out some feature that was not seen before. If we focus on the cognitive (or perceptual, in this case) act, we could say that a new perspective on the figure is created.

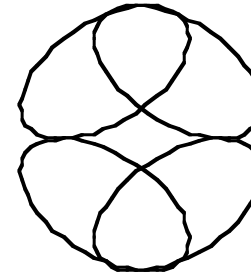

A

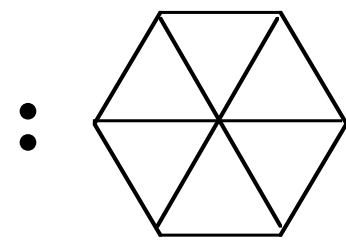

B

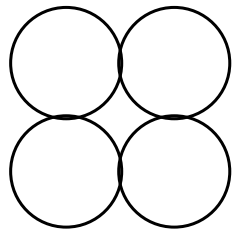

C

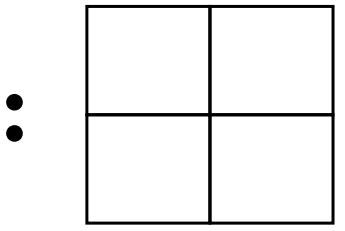

D

Fig. 2. A proportional analogy relation ( $A$ is to $B$ as $C$ is to $D$ ), in which the term $B$ is the same as in Fig. 1 . 
As another example, consider the T-puzzle with which some of you may be familiar. In this puzzle four wooden pieces are given (Figure 3) and the object is to arrange them in the shape of a ' $\mathrm{T}$ '.
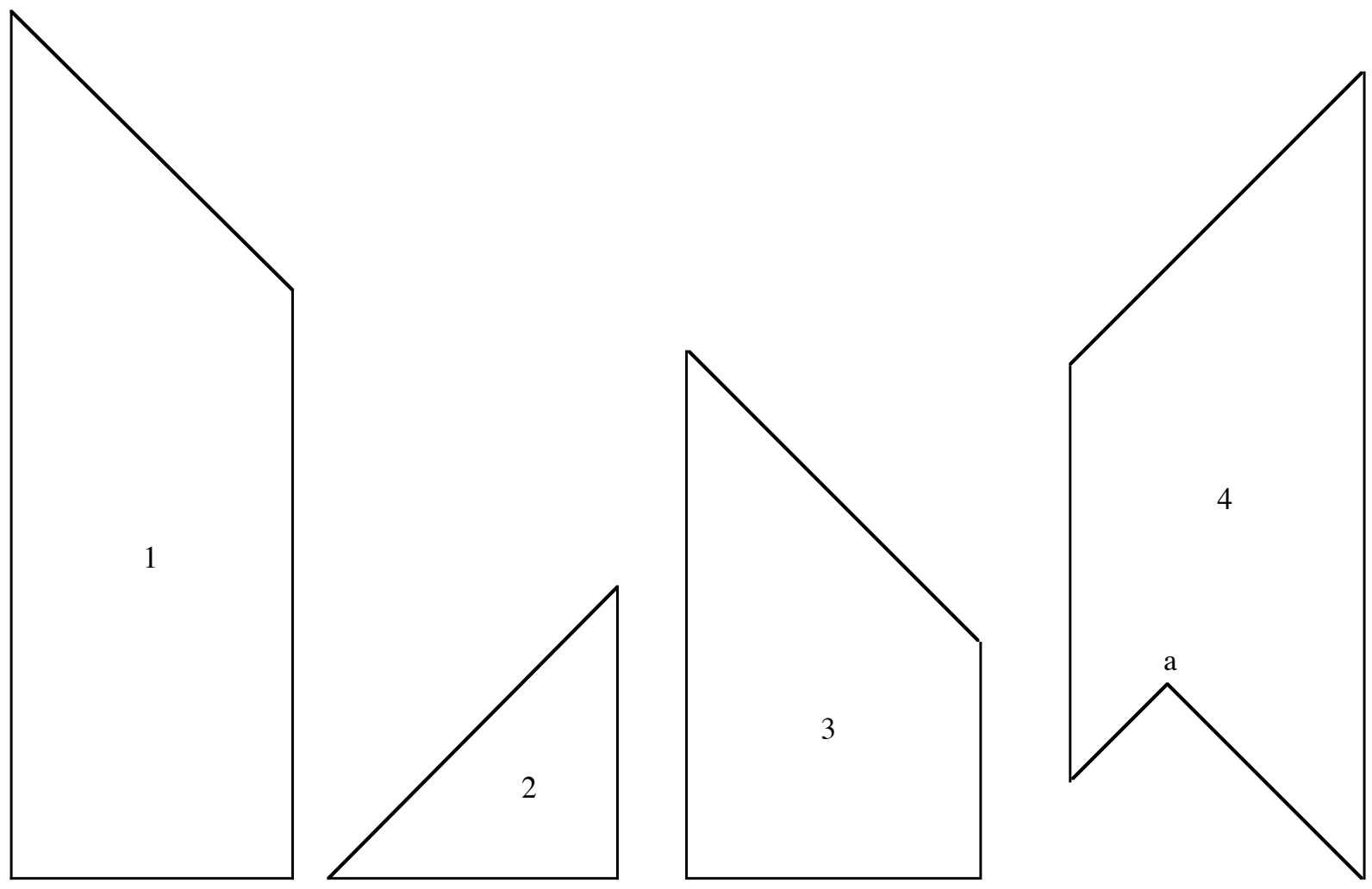

Fig. 3. T-puzzle. The objective is to arrange the given four pieces in the form of a T.

The puzzle seems quite simple at first: there are only four pieces and there are only so many ways in which they can be arranged. However, people can spend hours on it without getting to a solution. The trick in this puzzle is that most people make the assumption that the corner marked (a) in Figure 3 must be filled in, when in fact, in the solution of the puzzle (Figure 4), this is an outside corner. (See Suzuki and Hiraki 1997, for a detailed study of how people attempt to solve this puzzle.) Here a perceptual feature that seems very salient must be suppressed by the cognitive act. The creativity lies in looking at the pieces of the puzzle from a new perspective. 


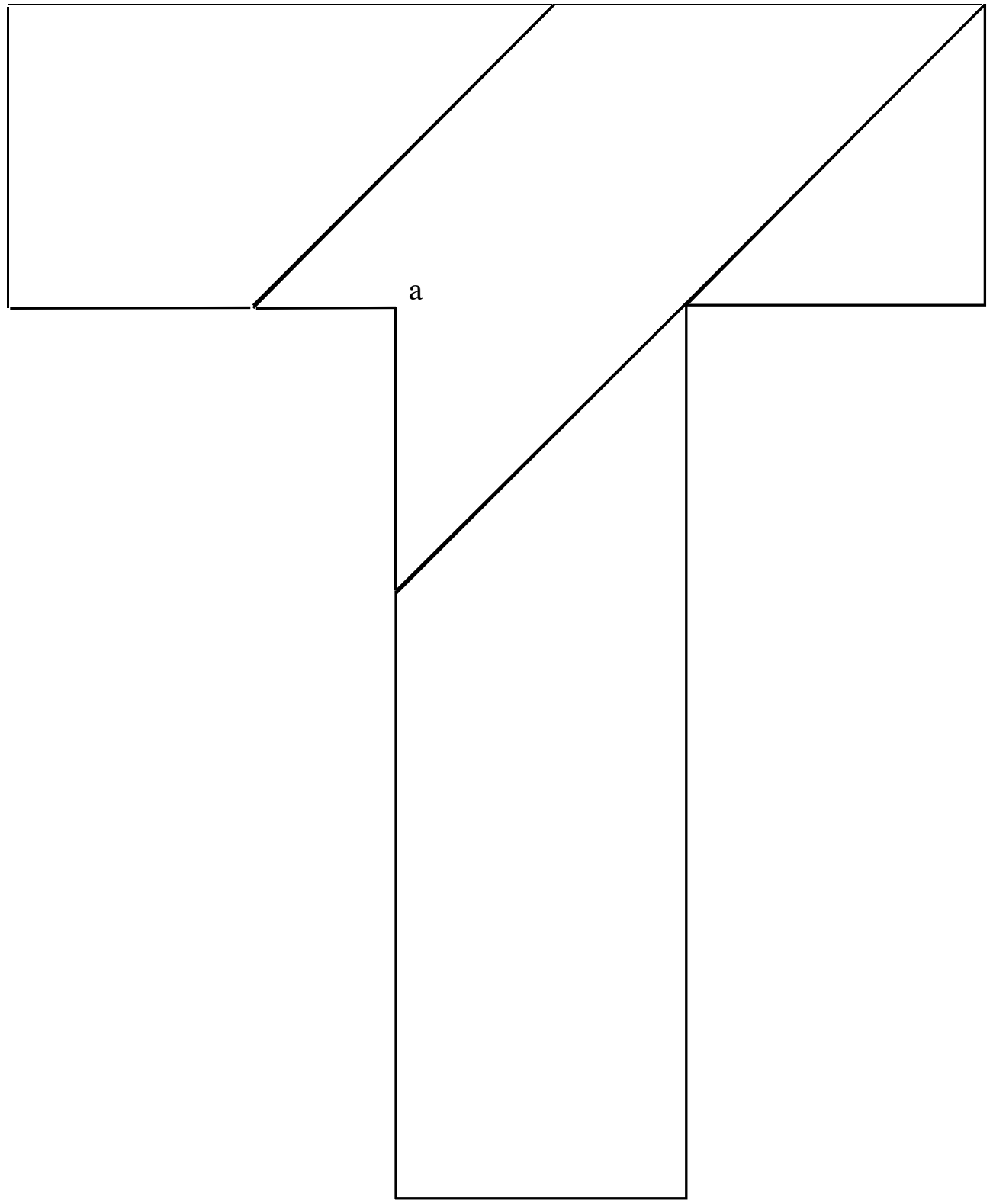

Fig. 4. Completed T-puzzle. Note that corner (a) is on the outside.

\subsection{Creativity in Conceptual Domains}

Let us now turn to other more 'conceptual' domains to see how creativity is manifested there. First consider problem solving, where creativity has an obvious application and hence has been studied by some scholars (Gordon 1961, Koestler 1964, Schön 1963). Perhaps the most striking example is provided by a case-study described in Schön (1963) where a product development team was faced with the problem of figuring out why synthetic-fiber paintbrushes were not performing as well as natural-fiber paintbrushes, and to improve their performance. The members of the team tried many ideas - for instance, they noticed that the natural fibers had frayed ends, and they tried to have synthetic fibers with frayed ends too - but without success. The 
breakthrough came when one member of the team suggested that the paintbrush might work as a pump. This idea was initially considered quite shocking, for a paintbrush and a pump were thought to be very dissimilar. Yet, in trying to make sense of the analogy, a new ontology and structure for the paintbrush was created. In this new representation, the paint was sucked in the space between the fibers through capillary action, and when the fibers were pressed against the surface to be painted, the curvature of the fibers caused a difference in pressure that pumped out the paint from the space between the fibers onto the surface to be painted. From this new ontology, when the synthetic-fiber and natural-fiber paintbrushes were compared, it was found that the synthetic fibers bent at a sharp angle against the surface, whereas the natural fibers formed a gradual curve. Thus, juxtaposition with pumping caused a new perspective to be created on the process of painting and paintbrush.

Moving to a very different domain, consider legal reasoning, something that many people may consider dry and formal, devoid of any creativity. Yet, there are often cases where using an analogy can create a completely new perspective (Hunter and Indurkhya 1998; Indurkhya 1997b). Take an example from a few years ago: Ben Johnson, an athlete, was given a lifetime suspension for testing positive twice for a banned substance. He appealed the lifetime suspension, his lawyer arguing, "A lifetime ban ... means, one talent that he has taken pride in, he can't use to make a living." The Athletic Federation's lawyer responded with an analogy, "It's a little like saying a jail sentence imposed on a jewel thief is a restraint of trade."

Needless to say, one major domain where creativity can be considered the life and blood is art and poetry. We show here how creativity in our sense is exemplified in poetry. Consider the following Japanese Haiku by a famous poet Jomei:

"Leaves of words,

autumn colors

a still mountain"

In this poem, words are compared with leaves and, by extension, colors of autumn on a mountain are compared to language. In understanding the poem, a new perspective is created on language and words. It is interesting to point out here that the Japanese character for language (言葉) is actually a compound of two characters, the first of which is 'words' (言) and the second is 'leaves' (葉). Nonetheless the metaphor has an element of surprise, and yields a perspective considered novel by most people. Such creation of novel features and perspective by juxtaposition is quite common in poetical metaphors (Gineste, Indurkhya and Scart 2000.)

\section{Cognitive Mechanisms of Creativity}

Over the past years some scholars have sought to extract cognitive mechanisms behind creativity and sometimes tried to formulate how they can be taught to people so as to enhance their creativity. These mechanisms can be generally divided into two classes, which we will refer to here as 'juxtaposition of dissimilar' and 'deconceptualization' respectively. We will now take a brief look at each in turn.

\subsection{Juxtaposition of Dissimilar}

This is known under various garbs: Gordon (1961) called it 'making the familiar strange', Schön 


\section{Bipin Indurkhya}

(1963) referred to it as 'displacement of concepts', de Bono (1975) named is as 'lateral thinking', and so on. But the basic mechanism is to juxtapose dissimilar concepts or objects so as to create new meanings and new perspectives through their synthesis. The underlying idea here is that if one recalls similar objects or concepts together, because they are already similar, their juxtaposition does not create any new meanings or perspective. However, putting dissimilar objects together forces one to reconsider the objects, and to stretch the imagination in order to find some meaningful connection. Whenever this process is successful, the resulting insights are often quite novel and striking. Famous poet Dylan Thomas, for example, described a similar mechanism that he claimed he used often while composing poetry.

The emphasis behind this mechanism is in moving away from similarity. This is a cognitively difficult task because our minds are full of associations, which are not easy to ignore. Hence various techniques like emptying the mind, random juxtaposition, etc. are taught as techniques to enhance one's creativity.

\subsection{Deconceptualization}

This is perhaps best illustrated using an example from Gianni Rodari's wonderful book The grammar of fantasy: An introduction to the art of inventing stories. In one of his techniques for creating new meanings (riddles, in this particular case) he offered a three-step process: 1) Estrangement: describe the object as though seen for the first time; 2) Association: the 'clear surface' of the description opens up the way for other meanings through images - so seek such images through associations; and finally 3) Metaphor: form a metaphor using the images from the last step. (Rodari 1996, p. 29.) The main objective here again is to move away from the existing conceptualization of the object, and the method focuses on somehow moving closer to the perceptual image of the object so that new associations may be found. Using this method, Rodari came up with the following new way of looking at a pen (in the form of a riddle): "What's black and needs white, to make its mark look bright".

One can find other examples of this mechanism in poetry. For example, in a classic poem Seascape by Stephen Spender, ocean is compared to a harp. Now this comparison seems very novel because ocean and harp are conceptually quite distinct objects in our language and culture. Yet, on reading the poem, one becomes aware of a perceptual resonance between the two: the light reflecting on the strings of a harp and the sunlight reflecting on the ocean waves, each forming a similar visual pattern. It is this perceptual association that carries the metaphor, and renders it meaningful.

It should be emphasized that the deconceptualization is also cognitively difficult in that it requires quite some cognitive effort to move closer to perception, and to see an object through perceptual images instead of via familiar conceptualizations. It should be noted in this connection that in learning to draw or paint, one must consciously 'learn' how to see blotches of colors and patterns of shadows instead of trees and fields, and to see lines and shapes instead of familiar faces. 


\section{Computational Modeling of Creativity}

\subsection{The Creative Potential of Computers}

Let us begin with some general observations on the creative potential of computers. Recall that we emphasized in the last section that the cognitive mechanisms of creativity - namely juxtaposition of dissimilar and deconceptualization - are difficult for us people because we are constrained by the associations of our concept networks that we inherit and learn in our lifetime. So it requires a significant amount of cognitive effort to break away from these associations.

Computers, on the other hand, do not have such conceptual associations. In fact, artificial intelligence research has spent a great deal of time and effort in modeling these conceptual associations - for they are a key to commonsense reasoning. Semantic networks, frames, scripts etc. are formalisms developed to capture this associativity. So, it follows that it must be easier for the computers to break away from the conceptual association than it is for the people. In other words, computers seem to be naturally inclined towards creativity.

Indeed, precisely this reasoning underlies the way in which computers are used to create art that, one could argue, transcends human art at Remko Scha's Institute of Artificial Art in Amsterdam (Harry 1992, 1997), as exemplified in the following passage:

"[I]t is practically impossible for human artists to create works of art that live up to the aesthetic ideals of philosophers like Immanuel Kant. Human artists always have rather selfish goals that usually involve money, fame and sex. Anyone who is aware of this, will become much too embarrassed to be able to engage in a disinterested process of aesthetic reflection. Machines are in a much better position to create objects of serene beauty; and computers will finally be able to create endless amounts of such objects, in infinite variety." (Harry 1997)

\subsection{Requirements for Computational Modeling of Creativity}

Let us now get more specific and consider how the cognitive mechanisms of juxtaposition of dissimilar and deconceptualization might be modeled computationally. First we must lay down the requirements that any computational system ought to have in order to be able to incorporate these mechanisms. These requirements are derived from our earlier work (Indurkhya 1992) on developing a model of cognition to explain how metaphors and analogies can create similarities. However, we believe that these requirements are quite reasonable and are met by many existing computational systems.

We require that there be multiple cognitive/perceptual layers with gradually increasing degrees of abstractions. For simplicity, we limit ourselves to two layers here: the perceptual layer contains less abstract perceptual, imagery, and episodic data; and the conceptual layer contains the more abstract conceptual representations. This is shown in Figure 5. We would like to note that while this requirement is met by many systems such as machine vision systems and speech recognition systems, there are also many other systems which do not explicitly recognize a hierarchy of different levels, and seek to explain creativity by mapping or some other such mechanism working within the same layer. 


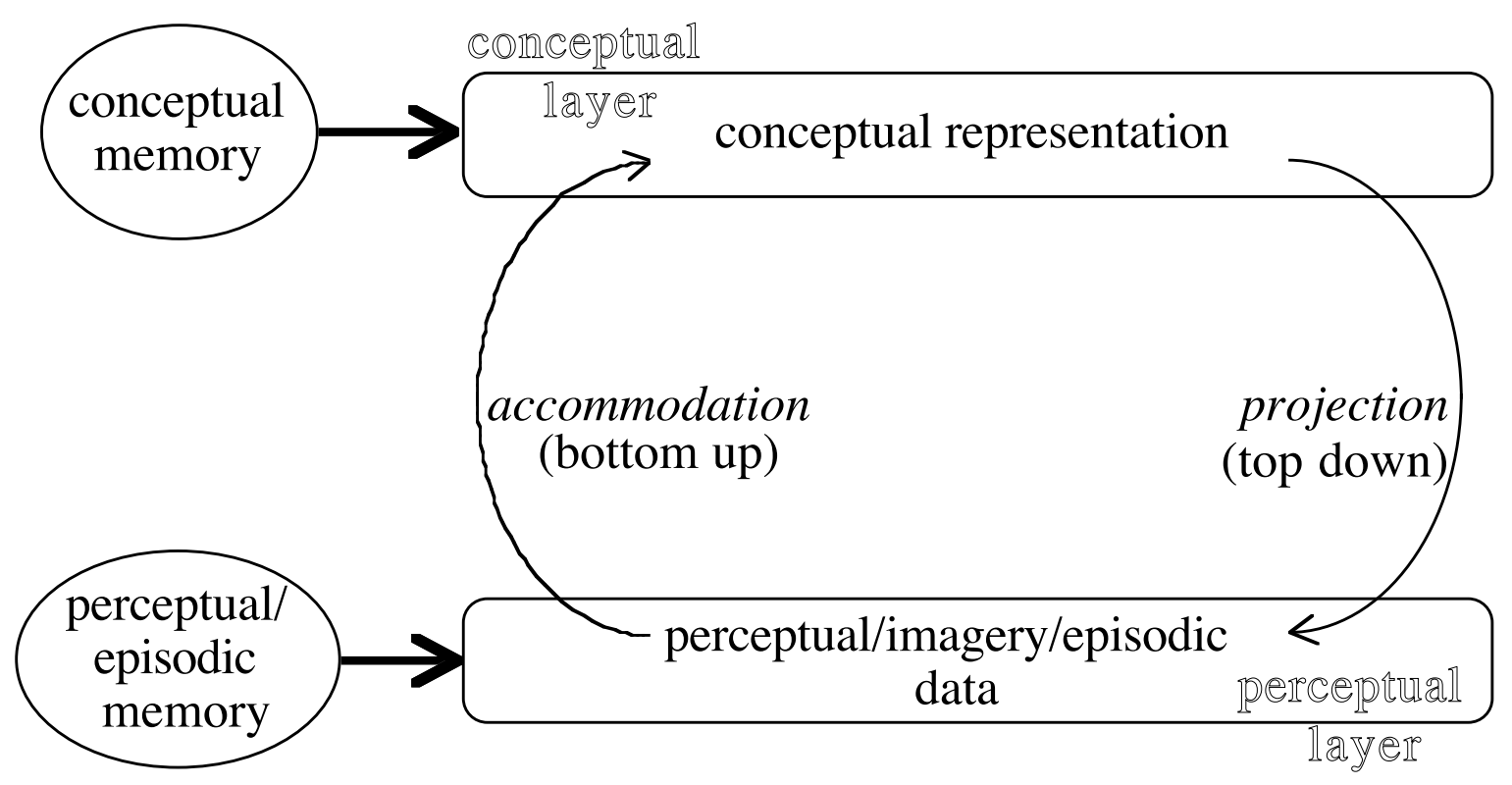

Fig. 5. A two-layered architecture for modeling creativity.

Secondly, we require top-down and bottom-up mechanisms working together to connect the conceptual representations to the perceptual data: we refer to these inter-level connections as interpretations. In our previous work, we have referred to the top-down mechanism as projection, and the bottom-up mechanism as accommodation. We emphasize again that in machine vision systems and speech recognition systems, such intermingling of top-down and bottom-up mechanisms is utilized to a great advantage.

Thirdly, we require that both the conceptual and the perceptual layers have their own autonomous structures. The structure of the conceptual layer reflects the conceptual associations that we normally acquire through our language and culture; and the structure of the perceptual layer reflects our prior perceptual experiences (episodes) with the objects. Note that this requirement presupposes an autonomous memory for each layer where the associations or structures of the respective layers are stored.

Fourthly, we assume that there are conventional interpretation relations between the two layers through which it is possible for concepts (which can be activated by words) to evoke perceptionlike images of the corresponding objects in the perceptual layer, and for the images in the perceptual layers to activate their corresponding concepts in the conceptual layer. Note that these conventional interpretations can be modeled as the default operations of the top-down and bottom-up operations of projection and accommodation. It should be emphasized that these inter-level activations are constrained by the autonomous structures of the respective memory modules. That is, when certain concepts evoke imagery in the perceptual layer, this imagery will be constrained by the prior perceptual experiences of the cognitive agent as reflected in the structure of the perceptual memory.

A crucial final assumption we make is that the image created in the perceptual layer (evoked by the concepts in the conceptual layer) can sustain itself and can be made available for 
reinterpretation. (It should be noted that this issue is somewhat controversial for some empirical research supports it while some other experimental work contradicts it. See for instance, Finke 1989; Logie and Denis 1991.)

\subsection{Modeling the Juxtaposition of the Dissimilar}

With the assumptions of our model clarified, we can elaborate on how 'juxtaposition of dissimilar' might lead to creative insights and how it might be modeled. This is graphically shown in Figure 6. Here we assume that one of the objects of juxtaposition is the focus of attention, which we refer to as the topic, for example the phenomenon of painting in the example of Schön mentioned above. However, it is also possible to have symmetric juxtapositions (as common in poetry and art), and we believe that our account can be extended to cover this case as well.

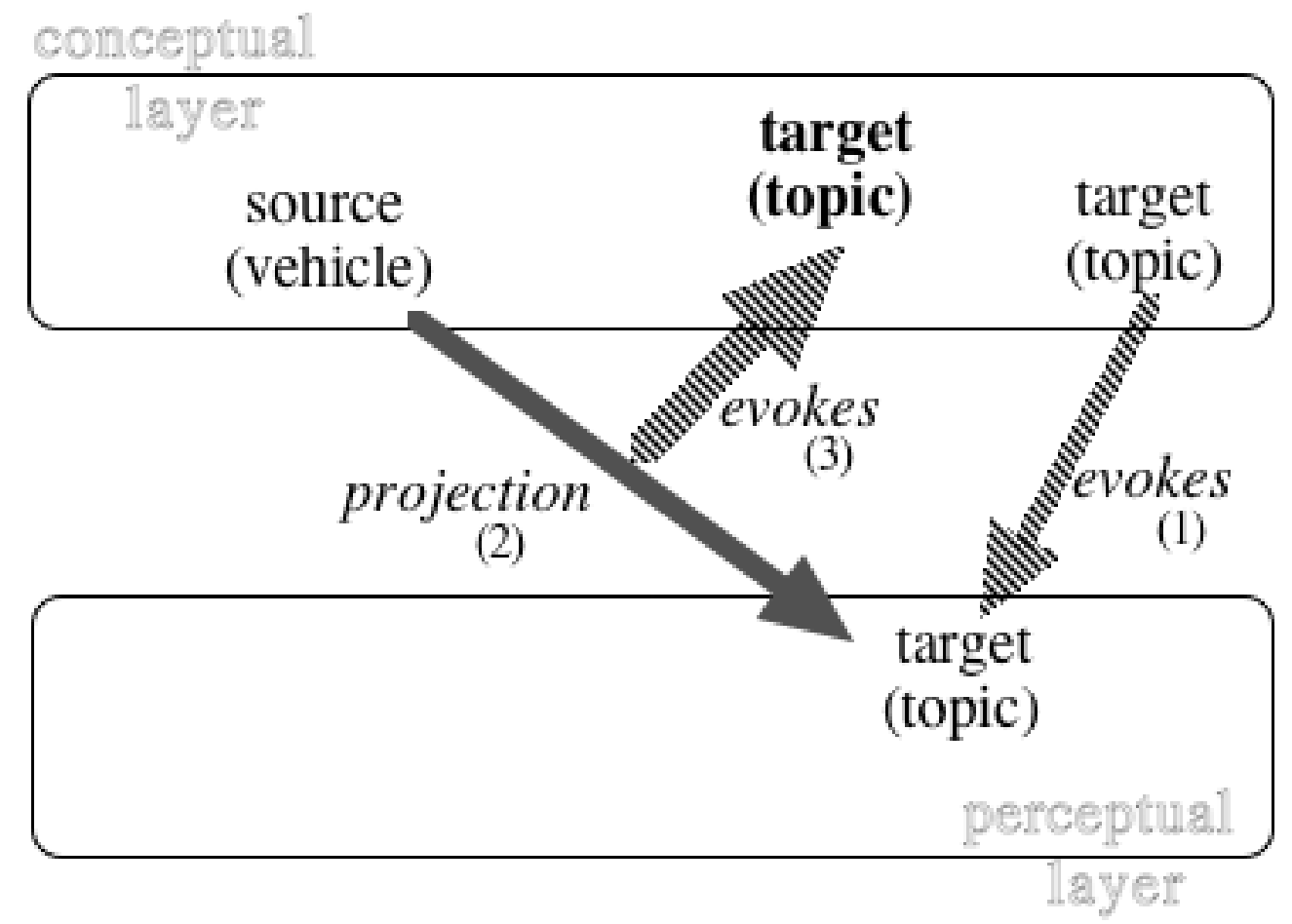

Fig. 6. Creative insights by 'juxtaposition of dissimilar'. The source concepts interact with the target image to create a new representation (with new features) of the target in the conceptual layer.

As shown in the figure, both the topic and the vehicle concepts are activated in the conceptual layer. The topic concepts evoke the imagery of the associated objects, and the vehicle concepts are projected on this imagery in a top-down fashion. As a result a new set of concepts get activated for the topic, thereby creating a new perspective on it.

Thus, we see that juxtaposition of dissimilar requires essentially modeling the top-down mechanism of projection. It is useful to point out that there are existing systems that do this kind of projection and can demonstrate 'creation' of features to some extent. For example, in a system 


\section{Bipin Indurkhya}

to detect man-made objects in satellite imagery (Mandal, Murthy \& Sankar 1996), if a certain part of the image is identified as an airport runway, conceptual features associated with the airport, like terminal buildings, car parking areas, etc. are projected onto the neighboring parts of the image. Similarly, in a speech recognition system, if the topic of conversation is known or identified, related concepts are projected onto the sound pattern (Erman et al. 1980).

There are also certain models specifically devoted to exploring this mechanism of creativity by juxtaposition. For example, Hofstadter and his colleagues (1995) have focused on simple but surprisingly rich domains such as letter strings and letter forms, and have implemented several systems to model how creative insights might be generated. Our own past work on modeling creativity of metaphor and analogy has been in this vein (Indurkhya 1992, 1997a), which has also been applied to legal reasoning (Indurkhya 1997b). Conceptual blending of Fauconnier and Turner's network model is yet another approach in this direction (Fauconnier and Turner 2002).

\subsection{Modeling 'Deconceptualization'}

How the mechanism of deconceptualization might be modeled is shown in Figure 7. Here, the source or the vehicle is not given. So the concepts of the topic are used to evoke an image of the associated objects. This image is then used to evoke an associated image at the perceptual layer. The associated image, in turn, activates its corresponding concepts, which together with the target concepts form a creative metaphor.

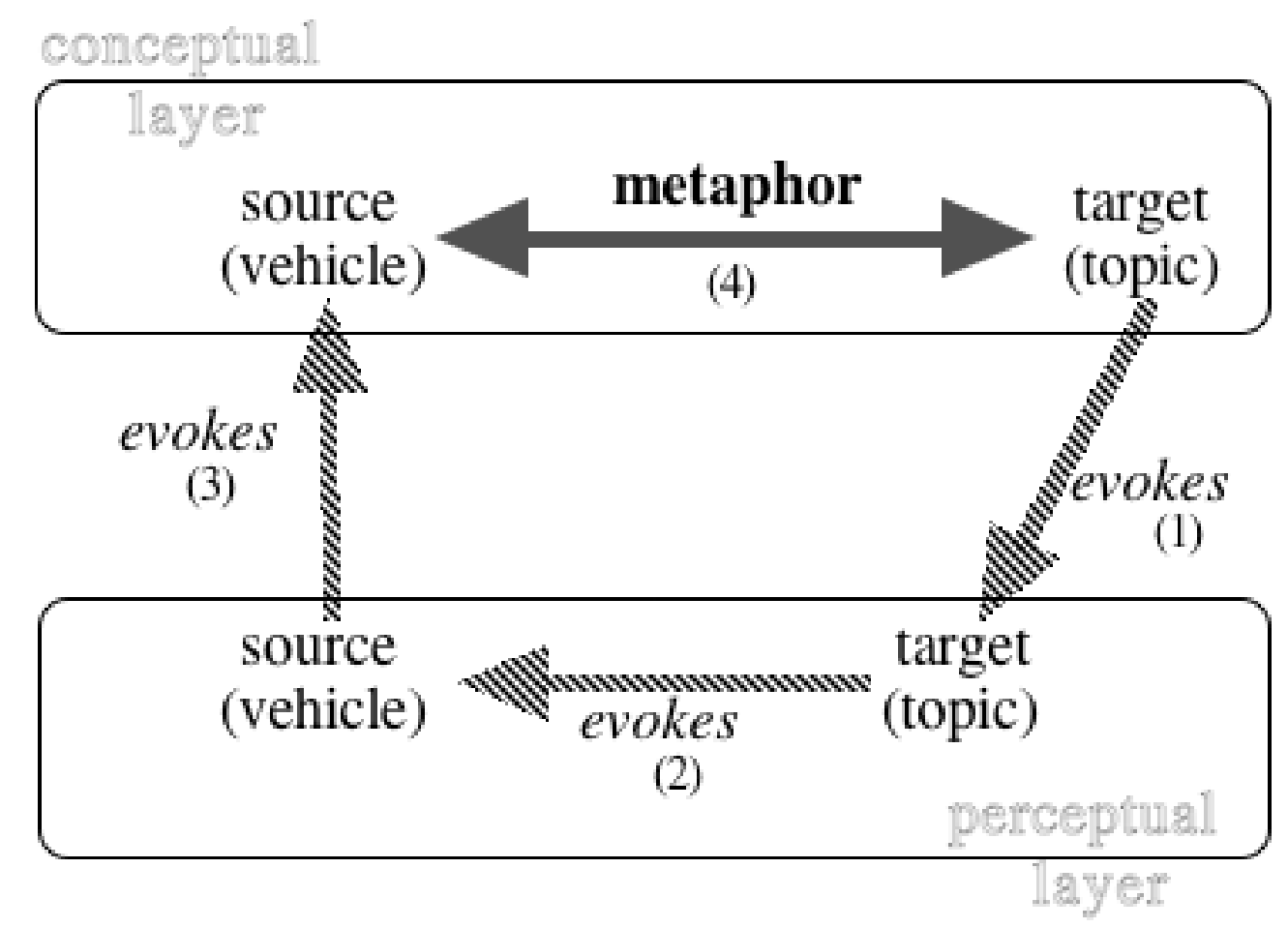

Fig. 7. Creative insights by 'deconceptualization'. The target image is used to evoke another image, which are then linked together as a metaphor in the conceptual layer. 
Notice that modeling this mechanism requires the bottom-up accommodation, and also some method for comparison at the perceptual/imagery level and an ability to form associations at the perceptual layer. Notice that this task may turn out to be no easier than that of encoding conceptual associations for a computational system, so a considerable work needs to be done in this direction.

\section{Conclusions and Future Research}

In summing up, we would like to reiterate what we believe are the key requirements for any computational system to be able to model creativity:

- to have a hierarchical model of cognition

- to integrate perception with cognition

- to incorporate imagery and episodic memory

- to have both top-down and bottom-up mechanisms for letting concepts interact with percepts.

We should mention one caveat though: we have not considered the purpose or utility of the cognitive act in our discussion on creativity. It is obvious that the purpose of the cognitive act plays an important role in how creativity is manifested in there, and it seems reasonable to suppose that different purposes might lead to different models of creativity.

Finally, we would like to reemphasize the main argument of this paper: For creativity a cognitive agent needs to free itself from the conventional conceptual associations. This task is cognitively difficult: the conceptual associations that one so dearly needs for all the commonsense reasoning can become a major stumbling block when it comes to creativity. But computers do not have such conventional associations; on the contrary, it is quite some work to program these associations into the computer. Therefore, we may conclude that computers are naturally pre-disposed towards incorporating creativity. This realization opens up a number of exciting possibilities to exploit the creative potential of computers in many different domains. We hope that the future research will utilize some of this potential in novel ways to enrich the human imagination and creativity.

\section{References}

de Bono, E. (1975). New Think: The use of lateral thinking in the generation of new ideas. Basic Books, New York.

Erman, L.D., Hayes-Roth, F., Lesser, V.R., and Reddy, D.R. (1980). The Hearsay-II speech understanding system: Integrating knowledge to resolve uncertainty. Computing Surveys 12, pp. 213-253.

Fauconnier, G., and Turner, M. (2002). The way we think: Conceptual blending and the mind's hidden complexities. Basic Books, New York.

Finke, R. (1989). Principles of mental imagery. MIT Press, Cambridge (Mass.).

Gineste, M.-D., Indurkhya, B., and Scart, V. (2000). Emergence of features in metaphor comprehension. Metaphor and Symbol 15, No. 3, pp. 117-135.

Gordon, W.J.J. (1961). Synectics: The development of creative capacity. Harper \& Row, New York.

Harry, H. (1992). On the role of machines and human persons in the art of the future. Pose 8 
(September 1992), pp. 30-35. Reprinted in P. Harmsen, E. Koppelman-Valk and M. Vredeling (eds.) Kunst en Technologie: The Beauty and the Beast? Eburon, Delft (1992), pp. $67-73$.

Harry, H. (1997). On the mechanism of human facial expression as a medium for interactive art. Catalogue Ars Electronica, September 1997, Linz, Austria.

Hunter, D. and Indurkhya, B. (1998) 'Don't think, but look' A Gestalt interactionist approach to legal thinking. In K. Holyoak, D. Gentner, and B. Kokinov (eds.) Advances in Analogy Research. New Bulgarian University, Sofia, Bulgaria, pp. 345-353.

Hofstadter, D., and The Fluid Analogies Research Group (1995). Fluid concepts and creative analogies. Basic Books, New York.

Indurkhya, B. (1992). Metaphor and cognition, Kluwer Academic Publishers, Dordrecht, The Netherlands.

Indurkhya, B. (1997a). Metaphor as change of representation: An artificial intelligence perspective. Journal of Experimental and Theoretical Artificial Intelligence 9, pp. 1-36.

Indurkhya, B. (1997b). On modeling creativity in legal reasoning. Proceedings of the Sixth International Conference on AI and Law, June 30 - July 3, 1997, Melbourne, Australia, pp. 180-189.

Koestler, A. (1964). The act of creation. Hutchinsons, London.

Logie, R.H., and Denis, M. (eds.) (1991). Mental images in human cognition. North-Holland, Amsterdam.

Mandal, D.P., Murthy, C.A., and Sankar, K.P. (1996). Analysis of IRS imagery for detecting man-made objects with a multivalued recognition system. IEEE Transaction on Systems, Man and Cybernetics - Part A: Systems and Humans, Vol. 26, No. 2, pp. 241-247.

Rodari, G. (1996). The grammar of fantasy. Teachers \& Writers Collaborative, New York.

Schön, D.A. (1963). Displacement of concepts. Humanities Press, New York.

Suzuki, H., and Hiraki, K. (1997). The constraint relaxation process as theory change: Toward theorizing the process of insight. (In Japanese.) Proceedings of the Japanese Cognitive Science Society's Special Interest Group on Language \& Learning, Vol. 97, No. 1, (SIGLAL 97-1), pp. 33-42. 